\title{
Criterios pedagógico-musicales que aplican directoras de coros para elegir repertorio en los coros infantiles
}
Pedagogical-musical criteria applied by choir directors to choose repertoire in children's choirs

\author{
Mariola Estefanie Godoy Díaz \\ mariolagodoy@gmail.com \\ Instituto de Música \\ Universidad Alberto Hurtado \\ Santiago, Chile \\ ORCID: https://orcid.org/0000-0002-8049-5773
}

doi: 10.7203/LEEME.48.20979

\section{Resumen}

El objetivo de investigación es identificar los criterios pedagógico-musicales que aplican directoras con experiencia en dirección coral infantil en la selección de repertorio porque existe una laguna en el conocimiento pedagógico sobre este tema. El método de este estudio cualitativo se enmarca en el diseño no experimental y transeccional, utilizando el método comparativo constante, puesto que se busca identificar solo mediante entrevistas los criterios pedagógico-musicales durante la elección de repertorio en coros infantiles. Los resultados indican que los criterios de selección de repertorio se basan en las inferencias que hacen las directoras sobre los gustos musicales del estudiantado, así como también en las dificultades y experiencias que cada corista exhibe. En conclusión, se identifican tres criterios pedagógicos en lo concerniente a la selección de repertorio: el bagaje musical, la dificultad del repertorio y los gustos musicales del estudiantado.

Palabras clave: Selección de repertorio; voces blancas; pedagogía coral; dirección coral; educación musical.

\begin{abstract}
The current research aims to identify music teaching criteria applied by women conductors who have experience in children's choral conducting because an existing gap in pedagogical in matters of repertoire selection. The research method holds a qualitative, nonexperimental, and cross-sectional design, using the constant comparative method because criteria are identified through conductor interviews that are centered on repertoire selection for childhood choirs. Results demonstrate that criteria for repertoire selection are based on inferences that conductors make regarding students' musical preferences and difficulties and experiences perceived by each singer. In conclusion, three teaching criteria are identified concerning repertoire selection: musical background, the difficulty level of repertoires, and musical tastes of students.
\end{abstract}

Key words: Repertoire Selection; Children's Voices; Choral Pedagogy; Choral Conducting; Musical Education. 


\section{Introducción}

Este estudio tiene como objetivo identificar los criterios pedagógico-musicales que aplican profesoras con experiencia en dirección coral infantil durante la elección de repertorio porque, existe un desconocimiento pedagógico en lo que concierne a la selección de repertorio para agrupaciones de voces blancas (Martín et al., 2018). Cabe señalar que la voz blanca se diferencia de la voz adulta por aspectos fisiológicos, ya que la laringe infantil es más corta que la de un adulto (Castro, 2018). Por lo tanto, no existen distinciones durante la infancia entre las voces femeninas y masculinas (Cáceres et al., 2019), lo que deja entrever que la selección de repertorio para un coro de voces blancas no respondería a los mismos criterios que se aplican para coros de voces adultas porque existen diferencias determinantes en cuanto a experiencia técnica, bagaje musical y aspiraciones artísticas (Brito, 2018; Vega, 2020).

Para alcanzar este objetivo se ha entrevistado a dos directoras que se han desempeñado en coros de voces blancas, siendo seleccionadas de manera deliberada porque cuentan con amplia experiencia en la dirección coral infantil, participando en reiteradas oportunidades en actividades corales de renombre, como, por ejemplo, el concurso Crecer Cantando del Teatro Municipal de Santiago.

\subsection{Criterios pedagógicos para la selección de repertorio en coros de voces blancas}

En los coros infantiles, se observa un desconocimiento pedagógico al momento de seleccionar repertorio apropiado para cada nivel educativo (Martín et al., 2018) considerando las particularidades de la voz blanca. Por ejemplo, Mozzoni et al. (2016) plantea que niños y niñas entre los 7 y 10 años podrían abarcar desde un Re a un Fa sobre la octava como extremos vocales, lo que podría variar según el grupo con el que cada director trabaje.

El concepto de voz blanca hace referencia a la voz de niños y niñas antes de la pubertad y se diferencia de las voces adultas, pues posee un registro agudo debido a que la laringe infantil es más corta (Castro, 2018). En esta etapa vocal, no existen diferencias entre las voces femeninas y masculinas, puesto que cantan en el mismo registro (Cáceres et al., 2019). Es por ello que la elección del repertorio a utilizar no debe ser una decisión superficial.

A la hora de seleccionar el repertorio, se han sugerido dos criterios. Por un lado, Carnassale (1995) se centra en la adquisición y desarrollo de la técnica, lo que implica que la educación musical sigue basándose en repertorios mal seleccionados sin equilibrar la técnica con el repertorio mismo. A partir de lo anterior, un equilibrio entre el repertorio y la técnica vocal, en el caso de un coro, podría mejorar la experiencia vocal en las escuelas, ya que mejoraría el sonido de grupo y brindarle al estudiantado una práctica coral sana vocalmente. En cuanto al canto en al 
aula, el Ministerio de Educación [MINEDUC] propone una progresión de Objetivos de Aprendizaje de Música de $1^{\circ}$ a $6^{\circ}$ básico: desde $1^{\circ}$ básico canto al unísono, desde $3^{\circ}$ básico canto al unísono y cánones simples, y desde $5^{\circ}$ básico canto al unísono y a más voces (MINEDUC, 2013a). Además, el canto sería una de las actividades musicales más importantes en la Educación Infantil y Primaria, ya que es una acción habitual en las aulas a través del tiempo (Muñoz, 2019).

Por otro lado, Verhagen et al. (2016) establecen que las necesidades musicales del estudiantado son un criterio para elegir el repertorio. Las posibles necesidades o preferencias de repertorio del estudiantado pueden variar, ya que estas preferencias están influidas por el entorno cultural en el que está inmerso el estudiantado (Almeida, 2016; Benhamu, 2017). Según la Segunda Encuesta Nacional de Participación y Consumo Cultural (2011), por ejemplo, los extremos de la brecha socioeconómica en Chile se posicionan, por una parte, en el sector con mayor ingreso, el que suele escuchar música rock, romántica, pop y música docta, en ese orden de preferencia. Mientras que, por otra parte, el sector con menores ingresos escucha música mexicana, música romántica, boleros y reggaetón, en ese orden de preferencia. Por lo expuesto, Huillipán y Angel-Alvarado (2020) mencionan que, para algunos/as docentes, el repertorio sugerido por instituciones educativas no es atingente a la realidad escolar.

Para aumentar el desarrollo musical y técnico en lo vocal, es fundamental que la elección del repertorio sea adecuada para generar en el alumnado una necesidad de adquirir nuevas destrezas y conocimientos musicales (Verhagen et al., 2016). Es decir, para elegir un repertorio, es necesario tener criterios de selección que sean atingentes y que permitan a los directores y directoras desarrollar una práctica coral adecuada con sus coristas. El MINEDUC recomienda utilizar repertorio que sea cercano en un comienzo y después ampliar a músicas de otros lugares geográficos (MINEDUC, 2013b), aproximando al alumnado desde lo conocido para posteriormente ir enriqueciendo la experiencia musical. En este sentido, una buena selección de repertorio, además de la técnica y cubrir las preferencias del alumnado, puede brindarle al estudiantado una experiencia musical y vocal que le permita conocer un mayor número de músicas distintas (Martín et al., 2018), ampliando sus conocimientos y enriqueciendo la experiencia vocal y musical del coro, incluso dando la posibilidad que el estudiantado manifieste propuestas de repertorio.

\section{Método}

El presente estudio tiene un enfoque cualitativo, porque se centra en identificar los criterios pedagógico-musicales aplicados por directoras de coros infantiles en cuanto a la selección de repertorio, mediante la recopilación de datos y analizando de forma comparativa los resultados (García, 2019). 


\subsection{Diseño}

El diseño de esta investigación es no experimental y transeccional, utilizando el método comparativo constante (García, 2019), puesto que se busca identificar solo mediante entrevistas los criterios pedagógico-musicales durante la elección de repertorio en coros infantiles. Este estudio se plantea desde un enfoque inductivo, ya que permitirá construir categorías y proposiciones teóricas basadas en los datos empíricos que arrojará la entrevista (Hernández, 2014).

\subsection{Objetivo de investigación}

El objetivo de esta investigación es identificar los criterios pedagógico- musicales que se aplican para elegir repertorio en los coros infantiles, con la finalidad de brindar un material académico en apoyo a todas aquellas personas que vayan a desempeñar esta actividad musical en escuelas por primera vez o en una etapa inicial. En cuanto a los objetivos específicos:

- Desvelar las percepciones que ha construido el profesorado sobre los coros de voces blancas.

Profundizar en los aspectos a considerar en la selección de repertorio para los coros de voces blancas.

Establecer la incidencia de las percepciones pedagógico-musicales que influyen en la elección de repertorio en torno a los coros de voces blancas.

\subsection{Contexto}

Generalmente, el coro de voces blancas dentro de las escuelas se lleva a cabo como una actividad extracurricular. Lo que significa que el estudiantado que participa en esta actividad musical lo hace fuera del horario de clases, por tanto, en un coro pueden existir integrantes de diferentes edades. En cuanto a la convocatoria a la participación, al coro se puede invitar a través de un afiche o cualquier actividad que visibilice la instancia. Para seleccionar al estudiantado, se puede filtrar mediante una audición considerando diferentes aspectos musicales (capacidades musicales, vocales o de interés), utilizando por lo general canciones del repertorio popular y de gusto personal (Castro, 2018). En caso de no optar por la selección, es posible integrar a todos los postulantes. Una vez completado el proceso de integración al coro, se comienza a buscar el repertorio a trabajar.

\footnotetext{
@ Mariola Estefanie Godoy Díaz. The content of this article is the sole responsibility of the authors. The Revista Electrónica de LEEME and Universitat de València are not liable for any legal actions that may arise involving the article's content. Revista Electrónica de LEEME - Lista Electrónica Europea de Música en la Educaciónhttp://ojs.uv.es/index/php/LEEME/index ISSN: 1575-9563. Editores: Universidad de Valencia y Jesús Tejada. Visibilidad de esta revista: SCOPUS, Emerging Sources Citation Index (Clarivate), EBSCO, CINDOC (CSIC), Citefactor, COPAC, Dialnet, DICE (CSIC), DOAJ, e-revistas (CSIC), EBSCO Premier, ERIH+, Gale Cengage Learning, IN-RECS, IRESIE, LATINDEX, MIAR, OCLC Worldcat, RESH, REDIB, RILM Core Journals, SUDOC, ULRICHS. Esta revista es de acceso libre mediante licencia Creative Commons 4.0 CC by. Política de archivado: etiqueta verde SHERPA-ROMEO.
} 


\subsection{Participantes}

El tipo de muestra para el estudio es no probabilística y deliberada, ya que las dos personas que han actuado como informantes responden a un determinado perfil. A saber, se desempeñan como docentes y directoras de coros infantiles, contando con más de 20 años de experiencia en la dirección de coros de voces blancas en la Región Metropolitana, en la ciudad de Santiago, así como también poseen una especialización en la dirección coral con escolares.

Específicamente, la Informante 1 es Licenciada en Educación Musical y directora de coros con 22 años de experiencia en la dirección de coros de voces blancas, habiendo participado varias ocasiones en el concurso Crecer Cantando, así como también en encuentros corales e incluso una gira con el coro infantil que dirige actualmente, el que se subdivide en tres coros. El primero comprende a estudiantes de primero a cuarto de Ed. Básica (6-9 años), el segundo a discentes de quinto a sexto de Educación Básica (10 y 11 años) y un tercer coro que reúne a estudiantes desde séptimo de Educación Básica cuarto curso de Educación Media (12-17 años). En cuanto a la recopilación de repertorio, la informante realiza la búsqueda por internet y ha tenido la oportunidad de mandar a hacer arreglos vocales para sus coros. Con respecto a la Informante 2, cabe señalar que es Profesora de Música, Educación Media y tiene un postítulo en Música con Mención en Dirección Coral. Cuenta con 31 años de experiencia en la dirección de coros de voces blancas, ha participado en el concurso Crecer Cantando en varias ocasiones y ha dirigido coros infantiles escolares y no escolares. Es preciso destacar su experiencia en la dirección de un coro inclusivo, puesto que participan niños/as y jóvenes con barreras psicomotoras y fonoaudiológicas, con quienes ha participado en instancias televisadas en el marco de campañas solidarias. La directora a lo largo de su vida ha recopilado repertorio de diferentes maneras, ya sea intercambiando o comprando partituras, ya sea encargando arreglos corales.

\subsection{Categorías}

Para llevar a cabo este estudio se establecieron dos categorías conceptuales:

- Criterios pedagógicos para el trabajo del coro.

- Tipo de repertorio.

Estas categorías permiten arrojar datos con el fin de llegar a una categoría central que dará respuesta a la pregunta de investigación. 


\subsection{Instrumento}

Una entrevista semiestructurada dirigida a la muestra que permite ahondar en los criterios pedagógico-musicales que se utilizan para seleccionar el repertorio (Anexo). Dicha entrevista fue validada por un panel de expertos conformado por un profesor de música especializado en métodos de educación musical, una directora de coros escolares ganadora del concurso "Crecer Cantando" y una socióloga y profesora de Música de Educación Media. Este panel de expertos revisó el instrumento de manera personal y enviaron comentarios para mejorarlo y revisar la atingencia del instrumento para el estudio. Los criterios de la entrevista se fundan en las decisiones pedagógicas que ha construido el profesorado sobre los coros de voces blancas (3 preguntas, por ejemplo: ¿Cómo selecciona al estudiantado?) y los tipos de repertorio que escoge el profesorado para los coros de voces blancas (4 preguntas, por ejemplo: ¿Qué criterios se utilizan para seleccionar el repertorio?). Las personas que participaron en la recolección de datos participaron en una entrevista privada y personal, la cual fue registrada mediante grabación audiovisual con previa autorización de las informantes.

\subsection{Procedimiento}

Las personas que fueron invitadas al estudio fueron contactadas mediante la plataforma WhatsApp donde se especificó la temática del estudio y el objetivo de la investigación. También, se les mencionó que su participación era mediante una entrevista semiestructurada confidencial, de las cuales la que tuvo mayor duración fue de 29 minutos y la de menor duración fue de 22 minutos. Además, se les informó al principio de las entrevistas que su participación sería privada, confidencial y que sería registrada en audio con previa autorización pudiendo el participante omitir información y/o poner fin a la entrevista si lo estimaba conveniente, esta información fue proporcionada mediante un libreto de consentimiento informado que expuso los códigos éticos de la actividad investigadora.

Tras la realización de las entrevistas, se han transcrito con el fin de realizar el análisis de datos, el cual se ha llevado a cabo mediante un proceso de codificación que permite construir un marco conceptual (Monge, 2015).

El análisis principalmente se hizo bajo la codificación abierta, axial y selectiva. Primero, la codificación abierta, la cual sirve para conceptualizar y codificar las ideas expuestas en cada una de las categorías conceptuales, segmentando estas ideas (San Martín, 2014). Segundo, la codificación axial permite buscar las posibles relaciones que se puedan establecer entre las dos categorías conceptuales (Palacios, 2016). Finalmente, la codificación selectiva, luego de establecer las relaciones entre las categorías y códigos, busca definir una categoría central que logre integrar los datos identificados en las etapas anteriores (Plaza, 2016). 


\title{
3. Resultados
}

\subsection{Criterios pedagógicos para el trabajo del coro}

Las directoras participantes no seleccionan a los coristas y la permanencia de dichos estudiantes se basa en actitudes, como la constancia en el tiempo y una asistencia frecuente a los ensayos. En cuanto a la deserción de estudiantes, ambas directoras enfatizan que la muda vocal, en el caso de los varones, es un factor determinante en el abandono de la práctica coral infantil. Respecto a la rutina de ensayo, la Informante 1 indica que:

\begin{abstract}
"Abordamos una partitura, la escuchamos un poco primero, vemos un poco el objetivo, vemos un poquito el compositor, qué quiso decir, como por dónde va y luego empezamos a leer las distintas líneas..., también ocupo harto la técnica de ir estudiando de atrás hacia adelante, es decir, si tengo una partitura de tres hojas veo primero la última sección”.
\end{abstract}

Aparte de lo musical, la Informante 1 también trabaja el área escénica de las presentaciones:

\begin{abstract}
"Ingresos del escenario, como subir a un escenario, como estar en un escenario, también es importante la parte escénica porque muchas veces yo le señalo a los alumnos que un coro no solamente se escucha, también se ve... nos preparamos no solamente en lo físico o en lo musical sino que también tiene que tener una cosa del modo de expresarlo, el controlar los nervios muchas veces si es que me pongo nervioso y en la medida que esté mejor ensayado y esté mejor sabido todo lo que tengo que hacer, es un poco más fácil controlar la parte escénica del momento o los nervios del momento".
\end{abstract}

En cambio, la Informante 2 expresa que se da un tiempo mínimo de ensayo de dos meses porque reconoce que, "cada coro es distinto. Como yo te decía, la mayoría de los niños que a mí me ha tocado, me ha tocado formarlos desde cero". En el caso de no poder tomarse ese tiempo mínimo, ella emplea un repertorio de baja dificultad durante los ensayos: "Música al unísono dependiendo de la cantidad de tiempo que llevaban los niños en el grupo. Si tenía mayoría de niños que llevaban más tiempo, entonces, ya podía empezar hablar de repertorio a más de una voz".

\subsection{Tipo de repertorio}

En el caso de ambas informantes, la octava es el límite para el ámbito que deben abarcar los repertorios para coros de voces blancas que vayan iniciando el trabajo vocal, especialmente cuando se trabaja con el alumnado más joven en los primeros niveles de Educación Básica. En este sentido, la Informante 1 manifiesta que:

"El caso de los más pequeños, generalmente deben ser canciones con un ámbito no tan grande, porque tú todavía no tienes técnicamente una voz muy desarrollada. Entonces, por ese lado tienes que buscar un repertorio que no tenga tanto ámbito, más o menos una octava y también 


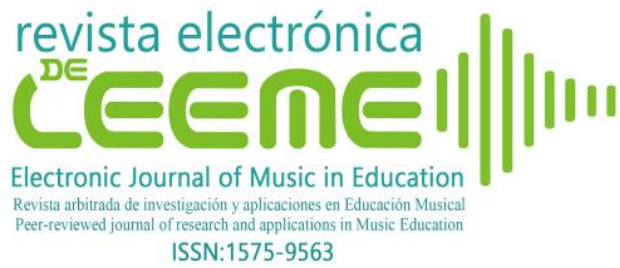

no muy grave, para no tener que trabajar tanto el registro grave, si no que inmediatamente instalar un poquito la voz "de cabeza". Por otro lado, también ojalá que las canciones tengan cierto movimiento para hacerlo más dinámico y que ellos no se cansen y se entretengan, casi como una especie de juego".

En la misma línea, la Informante 2 menciona que trabaja música con las siguientes características:

"Con los niños chiquititos, muy chiquititos, tienen que ser canciones infantiles. Hay muchas, muchas. Se parte con unísonos, obviamente, con un ámbito de la melodía que no sea muy amplio, generalmente inferior a una octava de diferencia entre la nota más aguda y la más grave".

Desde tercero o cuarto básico, las directoras trabajan repertorio que superan la octava. En este marco, la Informante 1 admite que:

\begin{abstract}
"Se entiende que debieran tener un poquito más de experiencia, por lo tanto, hay una dificultad mayor en términos musicales. Se pueden hacer algunas disonancias, puedes trabajar más altos, notas que digamos, partituras que ya puedan tener más saltos en sus intervalos que trabajan, más matices también y más expresión”.
\end{abstract}

Mientras que la Informante 2 menciona que:

"Ya no hay prácticamente problemas con la amplitud de... la tesitura de los niños y ahí ya tú puedes empezar a distinguir entre niños que tiene las voces más graves y las voces más agudas. Entonces, puedes empezar a pensar en trabajo más allá del canon en el sentido que en el canon cantan todos lo mismo. Cuando los niñitos ya son más grandes y se identifican las diferencias entre voces más graves y agudas, ya puedes empezar a hablar de partituras a dos voces, a tres voces con soprano primera, soprano segunda, contralto, etcétera".

El tipo de género musical que repitieron ambas informantes fue la música de películas. Por un lado, la Informante 1 menciona que "En el año 2004, existió una película que tuvo premio de película extranjera que fue Los Coristas. Fue una película en francés”. Ella propuso el repertorio en el coro y a lo largo de los años se fue manteniendo ese tipo de música en la agrupación a petición de los cantantes. Por otro lado, basándose en su experiencia la Informante 2 menciona que "a los niños les gustan las películas, les gusta las canciones de las películas, entonces, por ahí va la cosa”.

El montaje de repertorio es importante para los coros, ya que ambas informantes destacan el valor de la frecuencia con la que llevan a cabo los ensayos. Si bien la Informante 1 precisa que "tenemos ensayos con los coros una vez a la semana..., yo quisiera que ensayáramos mucho más"; la Informante 2 tiene en cuenta otros factores. Específicamente, señala la importancia de conocer qué tan responsables son los integrantes del coro para determinar la frecuencia de los ensayos, puesto que, a mayor responsabilidad, "pueden ser más frecuentes los ensayos y más largos”. En cambio, ante una menor actitud responsable, "hay que tratar de hacer ensayos cortitos y no muy frecuentes". 
En el montaje de repertorio, la Informante 1 busca darle mayor seguridad al coro mientras va cantando de la siguiente manera:

"Si tengo una partitura de tres hojas, veo primero la última sección, porque generalmente pasa que los coros se saben muy bien los comienzos, pero se vuelven inseguros cuando las obras son un poquito más largas para llegar al final. Entonces, es mucho mejor estudiar los finales... porque lo que te va pasando es que en el momento de cantarla el coro se siente cada vez más seguro".

En cuanto a recursos de apoyo en el montaje de repertorio, el uso de partituras es importante para las dos participantes, ya que ambas manifestaron el uso de la partitura como apoyo en los ensayos. La Informante 2 menciona lo siguiente:

"Los niños tienen muy buena memoria, mucho mejor que la de los adultos, entonces, para los que tiene memoria visual es muy buena la ayuda de la partitura. No es necesario que sepan solfear a primera vista, porque son muy pocos los coros donde la gente puede solfear a primera vista. Si es importante que aprendan a guiarse por la partitura".

Complementado lo anterior, la Informante 1 utiliza el apoyo de recursos auditivos:

"Obviamente abordamos una partitura, la escuchamos un poco primero, y se les manda audio por voces a sus papás por WhatsApp o por mail, para que puedan repasar en la casa, aunque no todos lo hacen, pero por lo menos está ese material de apoyo".

La Informante 2 usa el apoyo de otros recursos:

"Yo uso piano en los ensayos. Bueno, con los niños chicos, los unísonos hay que hacerlos con acompañamiento instrumental y de a poquitito uno va soltando también el acompañamiento instrumental y los va dejando más trabajar a capella, que es lo más difícil del canto coral: sostener la afinación cuando cantan a capella".

Para la selección misma del repertorio, la muestra toma en consideración los gustos musicales del estudiantado para cautivar o mantener la motivación de los estudiantes en la actividad coral. En el caso de la Informante 2, les solicita un listado de canciones para tomarlas en cuenta, pero ambas directoras filtran la música de las preferencias del estudiantado, siendo ellas quienes eligen qué cantar.

En cuanto a los criterios de selección de repertorio, la Informante 1 divide los repertorios en tres secciones de cara a un concierto: "una sección clásica, una sección folclórica y una sección popular. Claramente ellos [estudiantes] van a disfrutar más la sección popular". Según esto, ella admite que busca repertorios en que "la melodía principal se vaya turnando en las voces, de manera que en algún momento todos hagan la melodía principal y todos acompañen". En cambio, la Informante 2 menciona que "lo primero es obviamente conocer muy bien a los niños con los que trabajo, eso es súper importante", además afirma que: 
Generalmente, les pido a ellos que den un listado de canciones que les guste y me dedico a buscar arreglos o adaptar arreglos".

También, la Informante 2 agrega: "me he dado cuenta de que los niños, cuanto más chicos, son más proclives al contrapunto que a la armonía, cuando uno trata de hacer acordes. Para ellos es más natural el contrapunto". Lo anterior también es uno de sus criterios de selección, ya que la Informante 2 establece, en una primera etapa, el canto unísono, así como también "cánones, quodlibet, canciones a dos, tres voces ... cantos antifonales". Posteriormente, trabaja la armonía desde una textura homofónica, puesto que ya puede "clasificar voces agudas, voces graves, voces intermedias" y trabajar "a dos y tres voces".

\section{Discusión y conclusiones}

Los criterios pedagógicos que se utilizan en los coros de voces blancas para la selección del repertorio se pueden encauzar en dos dimensiones: lo cognitivo y lo emotivo. Desde la dimensión cognitiva, se establece que el repertorio seleccionado depende, por un lado, de la experiencia vocal del coro infantil para abordar el montaje de un repertorio y, por otro lado, de la dificultad técnico-musical que implica el repertorio para la agrupación. En otras palabras, desde la dimensión cognitiva, es importante que la persona que se desempeñe en la dirección conozca al grupo y las capacidades musicales de cada integrante, ya que las experiencias individuales podrían variar debido a la diversidad de edades y bagaje coral que puede existir entre cada estudiante y el coro como conjunto (cf. Almeida, 2016; Cáceres et al., 2017; Martín et al., 2018; Mozzoni et al., 2016; Vega, 2020,). Con respecto a la dimensión emotiva, se sostiene que la selección del repertorio se basa en los gustos musicales de los coristas con el fin de estimular el interés por la actividad coral y la permanencia en la agrupación, vinculando de esta manera al estudiantado con el proceso de selección del repertorio (Verhagen et al., 2016). En este punto, Almeida (2016) menciona que cuando los/as coristas participan en la selección del repertorio les motiva a permanecer en el coro y se sienten parte del grupo, de modo que las preferencias individuales se verían influenciadas por el entorno cultural (Benhamu, 2017). Cabe señalar que ambas dimensiones conviven en paralelo, tanto en el espacio del ensayo como en las presentaciones o conciertos, puesto que, según la teoría cognitivo-experiencial (Epstein, 1998), lo analítico y lo experiencial actúan en conjunto en la generación del comportamiento y el pensamiento.

Por lo expuesto, se identifican tres criterios pedagógicos (Figura 1). El primero tiene que ver con el bagaje musical y vocal del coro (Vega, 2020) porque es importante al momento de seleccionar el repertorio tener presente las diferentes experiencias musicales que podrían existir entre el estudiantado y la experiencia en común en la agrupación. El segundo se enmarca en la dificultad técnica-musical del repertorio (Carnassale,1995), porque es fundamental para la persona que dirija un coro saber detectar las posibles dificultades o limitaciones que se pueden 
presentar en un repertorio en particular, pensando en la agrupación en la que se esté trabajando. Por último, el tercer criterio pedagógico se centra en los procesos de selección del repertorio porque, mientras se observa en las informantes que la elección se basa en el gusto estudiantil según la perspectiva adulta, es preciso transitar hacia una selección participativa, en donde el estudiantado tenga derecho a opinar y a escoger repertorios (Verhagen et al., 2016) para favorecer el descubrimiento y la socialización de repertorios de una manera transversal y horizontal (Martín et al., 2018).

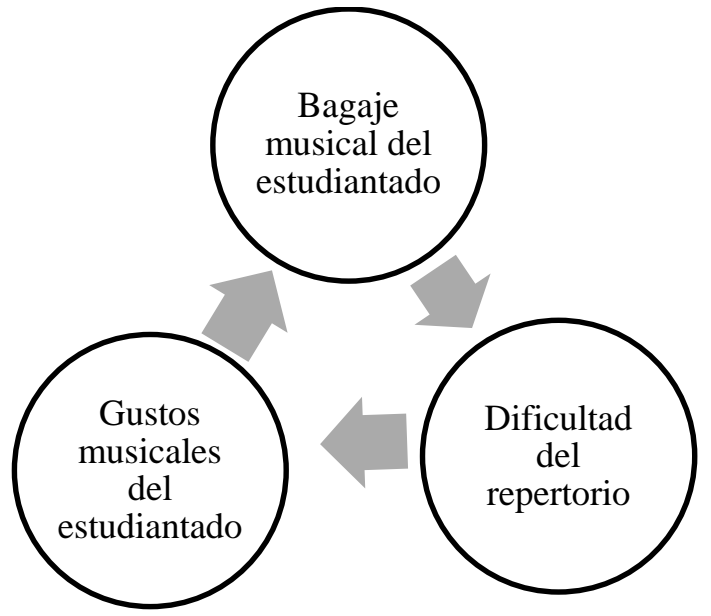

Figura 1. Criterios pedagógico-musicales empleados en coros de voces blancas

Se han identificado tres criterios pedagógico-musicales que se aplican para elegir repertorio en los coros infantiles. El primer criterio concierne al bagaje musical y vocal del estudiantado, puesto que se funda tanto en las experiencias personales de cada estudiante como en la experiencia colectiva dentro de la agrupación. El segundo criterio corresponde a la dificultad del repertorio, ya que cobran relevancia los elementos técnico-musicales que plantean desafíos musicales al grupo de cantantes, tanto en su individualidad como en el trabajo de ensamble. Por último, el tercer criterio se vincula a los gustos musicales del estudiantado porque favorece el interés y la permanencia del estudiantado por la actividad coral. Por todo lo dicho, se concluye que los tres criterios descritos interactúan transversalmente a la hora de seleccionar un repertorio para coro de voces blancas, ya que abarcan diferentes aspectos atingentes a un coro infantil. Esta interacción está basada en el bagaje musical del alumnado porque tal criterio, de una u otra manera, permite comprender la subjetividad que poseen los niveles de dificultad técnico-musical y los gustos musicales, dado que el bagaje hace referencia a una trayectoria musical que da cuenta de un proceso de construcción personal que afecta en lo colectivo, manifestándose a través de los gustos y de los niveles de dominio técnico. 
Por todo lo dicho, surgen al menos tres implicaciones. Primero, desde una perspectiva teórica es necesario profundizar en los gustos musicales del estudiantado de Educación Básica, ya sea a nivel comunal, regional o nacional. Segundo, desde una perspectiva práctica, queda en evidencia la necesidad de formar al profesorado en la dirección coral infantil, particularmente en el ámbito de la elección de repertorio para voces blancas a través de instancias de seminarios o cursos complementarios. Por último, desde una perspectiva pedagógica se propone hacer parte al estudiantado de los procesos de construcción curricular, promoviendo practicas constructivistas que favorezcan comportamientos de orden activo y participativo. Es decir, que el alumnado tenga capacidad de decisión y su participación no se reduzca a proponer repertorios para el trabajo coral.

\section{Referencias}

Almeida, M. (2016). Escolhendo o repertório coral: uma tarefia de regentes? Revista Música Hodie, 16(2), 25-34, https://www.doi.org/10.5216/mh.v16i2.45212

Benhamu, T. (2017). Factores influyentes en las preferencias musicales de los alumnos de Educación Primaria. (Tesis de Licenciatura). Universidad de Sevilla. https://idus.us.es/handle/11441/62890

Brito, D. (2018). Do Canto Orfeônico às práticas corais infantis: o desenvolvimento histórico do Canto Coral no Brasil. En XXIII Congresso Nacional da Associação Brasileira de Educação Musical, Educação Musical en tempos de crise: percepções, impactos e enfrentamentos. Santa Maria/RS- 26 a 28 de septiembre de 2018. http://abemeducacaomusical.com.br/conferencias/

Cáceres, N., Flores, M., Hernández, E. y López, R. (2019). Propuesta de un repertorio de canciones y actividades para el correcto trabajo de las voces blancas en alumnos de primer ciclo básico. (Tesis de Licenciatura). Universidad Andrés Bello. http://repositorio.unab.cl/xmlui/bitstream/handle/ria/

Carnassale, G. (1995). O ensino de canto para crianças e adolescentes. (Tesis de Licenciatura). Universidade Estadual de Campinas. http://repositorio.unicamp.br/bitstream/REPOSIP

Castro, L. (2018). Importancia de la experiencia metodológica en la enseñanza de los coros de voces blancas. Revista de Investigación y Pedagogía del Arte, 4, 1-7. https://publicaciones.ucuenca.edu.ec/ojs/index.php/revpos/article/view/2149

Consejo Nacional de la Cultura y las Artes (2011). Segunda Encuesta Nacional de Participación y Consumo Cultural (ENPCC). Ediciones Cultura. https://www.cultura.gob.cl/wp

Epstein, S. (1998). Constructive thinking: the key to emotional intelligence. Praeger. 
García, P. (2019). El método comparativo constante y sus potencialidades para el estudio de políticas educativas para la escuela secundaria en Latinoamérica. Revista Latinoamericana de Educación Comparada, 10(15), 27-43. https://dialnet.unirioja.es

Hernández, R. (2014). La investigación cualitativa a través de entrevistas: su análisis mediante la teoría fundamentada. Cuestiones Pedagógicas, 23, 187-210. https://revistascientificas.us.es/index.php/Cuestiones-Pedagogicas/article/view/9815

Huillipan, J. y Angel-Alvarado, R. (2020). Arreglos musicales en el aula: Factores pedagógicos en la Educación Primaria. Revista Electrónica LEEME, 45, 53-67. https://doi.org/10.7203/LEEME.45.16527

Martín, M. Velázquez, L. y Reyes, M. (2018). La utilidad del repertorio coral. Apuntes para una historia. Revista Electrónica Luz, 17(Especial), 45-53. https://luz.uho.edu.cu/index.php

Ministerio de Educación (2013a). Anexo. Progresión de Objetivos de Aprendizaje para música de $1^{\circ}$ a $6^{\circ}$ básico. En Bases Curriculares de primero a sexto básico. MINEDUC. https://www.curriculumnacional.cl/portal/

Ministerio de Educación (2013b). Programa de estudio quinto año básico. MINEDUC. https://www.curriculumnacional.cl/614/articles-20710_programa.pdf

Monge, V. (2015). La codificación en el método de investigación de la Grounded Theory o Teoría $\begin{array}{llll}\text { Fundamentada. Innovaciones } & \text { Educativas, }\end{array}$ https://www.doi.org/10.22458/ie.v17i22.1100

Mozzoni, G., Santana, A., Sayago, A., Toledo, M., Martínez, P., Sagrera, M. y Rozenvit, M. (2016). Estudio sobre la extensión vocal en niños de 7 a 10 años. Revista de Investigaciones en Técnica Vocal, 4(1), 53-66. https://dialnet.unirioja.es/

Muñoz, R. (2019). ¿A quién le importa el canto en el aula? Estudio basado en un cuestionario. Revista Electrónica LEEME, 44, 1-23. https://www.doi.org/10.7203/LEEME.44.15631

Palacios, G. (2016). La codificación Axial, innovación metodológica. Revista Electrónica Científica de Investigación Educativa, 3(1), 497-509. https://www.rediech.org/

Plaza-Galvez, L. (2016). Obstáculos presentes en Modelación Matemática. Caso Ecuaciones Diferenciales en la formación de Ingenieros. Revista Científica, 2(25), 176-187. https://www.doi.org/10.14483//udistrital.jour.RC.2016.25.a

San Martín, D. (2014). Teoría fundamentada y Atlas.ti: recursos metodológicos para la investigación educativa. Revista Electrónica de Investigación Educativa, 16(1), 104-122. https://www.redalyc.org/pdf/155/15530561008.pdf 


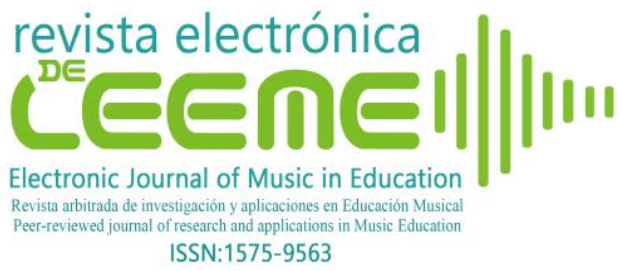

Vega, P. (2020) Comprendiendo la experiencia musical en el currículum musical chileno. Revista Latinoamericana de Filosofía de la Educación, 7(14), 203-217 http://ixtli.org/revista/index.php/ixtli/article/view/141

Vera, A. (2018). Editorial. Resonancias, 22 (43), 9-11. http://resonancias.uc.cl/

Verhagen, F., Panigada, L. y Morales, R. (2016). El Sistema Nacional de Orquestas y Coros Juveniles e Infantiles de Venezuela: un modelo pedagógico de inclusión social a través de la excelencia musical. Revista Internacional de Educación Musical, 4, 35-46. https://www.doi.org/10.12967/RIEM-2016-4-p035-046

@ Mariola Estefanie Godoy Díaz. The content of this article is the sole responsibility of the authors. The Revista Electrónica de LEEME and Universitat de València are not liable for any legal actions that may arise involving the article's content. Revista Electrónica de LEEME - Lista Electrónica Europea de Música en la Educación-. http://ojs.uv.es/index/php/LEEME/index ISSN: 1575-9563. Editores: Universidad de Valencia y Jesús Tejada. Visibilidad de esta revista: SCOPUS, Emerging Sources Citation Index (Clarivate), EBSCO, CINDOC (CSIC), Citefactor, COPAC, Dialnet, DICE (CSIC), DOAJ, e-revistas (CSIC), EBSCO Premier, ERIH+, Gale Cengage Learning, IN-RECS, IRESIE, LATINDEX, MIAR, OCLC Worldcat, RESH, REDIB, RILM Core Journals, SUDOC, ULRICHS. Esta revista es de acceso libre mediante licencia Creative Commons 4.0 CC by. Política de archivado: etiqueta verde SHERPA-ROMEO. 


\section{Anexo: Entrevista semi-estructurada.}

\section{Datos del docente entrevistado.}

Edad:

Formación académica:

Lugares de formación:

Años que se desempeña como director/a de coros infantiles:

Años que se desempeña como director/a en el coro actual:

\section{I.- Criterios pedagógicos para el trabajo del coro.}

A. ¿Cómo selecciona al estudiantado?

B. ¿Existe algún requisito de permanencia? ¿Cuáles son esos requisitos?

C. ¿Cuál es la rutina de preparación de cara a un concierto?

D. ¿Me podría compartir algunos hitos artísticos del coro?

\section{II.- Tipos de repertorio.}

A. ¿Qué criterios educativos se utilizan para seleccionar el repertorio?
A.1. ¿Cómo consigue el repertorio?
B. ¿Cómo suele trabajar el montaje de repertorio?
C. ¿Existe algún repertorio que permanezca en el tiempo? De ser así, ¿Por qué? 